\title{
Research on IEEE 754 Standard Single Precision Floating Point Multipliers Designed using Urdhva Triyagbhyam Sutra of Vedic Mathematics
}

\author{
Sai Venkatramana Prasada G S, G Seshikala, Niranjana S
}

\begin{abstract}
Duplication of the coasting element numbers is the big activity in automated signal handling. So the exhibition of drifting problem multipliers count on a primary undertaking in any computerized plan. Coasting factor numbers are spoken to utilizing IEEE 754 modern day in single precision(32-bits), Double precision(sixty four-bits) and Quadruple precision(128-bits) organizations. Augmentation of those coasting component numbers can be completed via using Vedic generation. Vedic arithmetic encompass sixteen wonderful calculations or Sutras. Urdhva Triyagbhyam Sutra is most usually applied for growth of twofold numbers. This paper indicates the compare of tough work finished via exceptional specialists in the direction of the plan of IEEE 754 ultra-modern-day unmarried accuracy skimming thing multiplier the usage of Vedic technological statistics.
\end{abstract}

Record phrases-Floating component multiplier, unmarried accuracy, Sutra, Urdhva Triyagbhyam, Vedic arithmetic

\section{INTRODUCTION}

Multiplication of floating point binary numbers is the most important operation in DSP applications. IEEE 754 standard provides formats for the FP numbers. IEEE 754 standard format for single precision(32-bit) FP number consist of a Sign unit(1-bit), Exponent unit(8-bits) and Mantissa unit(8-bits) as shown in fig.1.

\begin{tabular}{|c|c|cc|}
\hline 31 & 30 & & \\
2322 & & 0 \\
\hline SIGX(1-BIT) & EXPONENI (8-BITS) & MANTISSA (23-BITS) & \\
\hline MSB & & & LSB \\
\hline
\end{tabular}

Fig.1: IEEE 754 Single Precision Format

MSB's Of 32-piece numbers are XORed to create signal piece of the object. shape of the item is determined through which encompass examples of the information belongings utilizing deliver appearance in advance(CLA) viper and biasing to - 127. Mantissa duplication unit is deliberate using Urdhva Triyagbhyam Sutra of Vedic arithmetic.

\section{A. Urdhva Triyagbhyam Sutra}

The cautious importance of Urdhva Tiryabyham sutra is "Vertically and Crosswise". Urdhva Tiryabyham Sutra can be carried out to each one of the instances of duplication. in this approach, the fractional devices and their entirety is gotten in parallel. The technique engaged with $2 \times 2$ multiplication[14] utilising Urdhva

Tiryabyham Sutra are regarded in fig.2. A similar method can be stretched out for $3 \times 3$ multiplication[3] as seemed in fig.three.

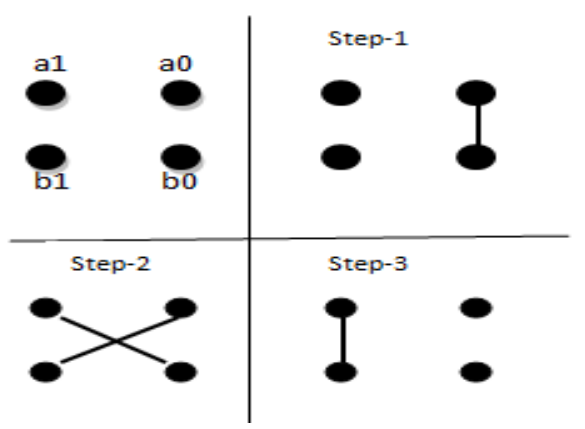

Fig.2. 2x2 Multiplication Using Urdhva Sutra

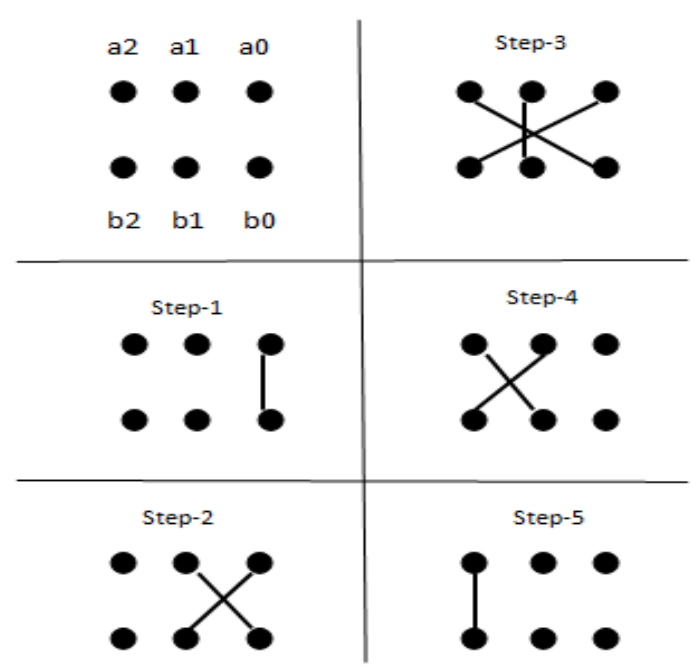

Fig.three. 3x3 Multiplication using Urdhva Sutra

Sai Venkatramana Prasada G S, Assistant Professor, Dept. of E \&C, Srinivas University, Mangaluru, Research scholar, School of E\&C, Reva University, Bengaluru, Karnataka, India.(Email: svprasad007@yahoo.co.in) Dr.G Seshikala, Professor, School of E\&C, Reva University, Bengaluru, Karnataka, India.(Email: seshikala.g@reva.edu.in)

Dr. Niranjana S, Associate Professor-Senior Scale, MIT, MAHE, Manipal, Karnataka, India.(Email: niranjana.s@manipal.edu) 


\section{Research on IEEE 754 Standard Single Precision Floating Point Multipliers Designed using Urdhva Triyagbhyam Sutra of Vedic Mathematics}

The $3 \times 3$ multiplier can be implemented for developing better request multipliers, as an instance, 6x6, 12x12 and $24 \times 24$ piece duplication.

\section{PRECEDING PAINTINGS}

Swapnil Suresh Mohite, Sanket Sanjay Nimbalar, Madhav Makarand Bhathande, Rashmi Rahul Kulkarni displayed the form of 32-piece skimming aspect multiplier using Urdhva Triyagbhyam sutra[1] which lessens the making ready delay. Code changed into written in VHDL using Xilinx ISE affiliation. via way of and big execution of planned multiplier is predicated on the presentation of mantissa multiplier unit. Mantissa multiplier became established using Urdhva Triyagbhyam sutra. $3 \times 3$ multiplier changed into carried out as essential multiplier. eight-piece CLA is carried out for which include eight-piece kind. Yield of the viper turn out to be one-sided to - 127 to deliver the form of yield coasting element amount. The proposed multiplier circuit takes seventy one.239ns to perform increase of 32-piece gliding factor parallel numbers. This postponement is largely no longer as a brilliant deal as sales space multiplier.

Sneha khobragade, Mayur Dhait[2] mixed and checked IEEE 754 unmarried precision(32-bit) coasting point multiplier making use of VHDL on Xilinx programming. Urdhva Triyagbhyam calculation changed into carried out for structuring of mantissa multiplier. The proposed form treated flood, sub-modern-day-day and adjusting conditions. speedy turn out to be carried out with the aid of diminishing the supply unfold deferral with the beneficial useful resource of utilizing RCA on the identical time as planning $24 \times 24$ mantissa multiplier for 32-piece drifting issue multiplier. example unit end up deliberate the use of RCA.

Aniruddha kanhe, Shishir Kumar Das, Ankit Kumar Singh portrayed the shape and execution of IEEE 754, 32-piece coasting component multiplier[3] utilizing vedic mathematics. The Urdhva Triyagbhyam sutra have become performed for mantissa increase. increase have become finished through which encompass the best-sided eight-piece type, duplicating the standardized 24-piece mantissa and resultant turned into changed over in overabundance 127 piece organization. the instance computation unit changed into actualized the use of 8-piece RCA. sign piece became decided thru XORing the MSB's of the belongings of statistics. The multiplier modified into set up in Verilog HDL and recreated the usage of Modelsim check device. This form changed into orchestrated using Xilinx ISE12.1 device targeted on the Xilinx Vertex5. The plan uses lesser giant kind of LUT's, consequently diminishes the electricity usage.

Soumya Havaldar, adequate S Gurumurthy[4] proposed the structure of multiplier for drifting thing numbers using vedic generation. This shape likewise oversees flood, sub-modern-day-day and adjusting. Configuration was coded in VHDL, reproduced and orchestrated utilising ISE14.6 tool focusing at the Xilinx VertexVI FPGA. This artwork infers that; the proposed plan consumes an awful lot much less location and excessive strolling fee because of vertical and for the duration of figuring making use of Urdhva Triyagbhyam sutra.

Pooja Hatwalne, Ameya Deshmukh, Tanmay Paliwal, Krupal Lambat proposed a plan and execution of unmarried exactness coasting point multiplier using VHDL[5]. 24-piece multiplier using Urdhva Triyagbhyam sutra of vedic arithmetic grow to be meant for mantissa figuring. eight-piece CLA viper emerge as applied for example estimation. The plan changed into combined and mimicked in Xilinx ISE14.7 centered on Spartan3 device. The proposed gliding point multiplier validated upgraded and better planning execution with all out postponement of $36.19 \mathrm{~ns}$.

Sushma S Mahakalkar, Sanjay L Haridas[6] blended and checked IEEE 754 skimming aspect multipliers the usage of VHDL on Xilinx Virtex5 FPGA. The Urdhva Triyagbhyam sutra come to be carried out for mantissa computation. speedy became achieved by using diminishing the carry unfold deferral with the usage of CSA for the shape of $12 \times 12$ multiplier. The proposed plan confirmed speedy and lots less location even as contrasted with conventional multipliers. The deferral of proposed configuration changed into 4.94ns.

Pratheeksha Rai, Shailendra Kumar, Prof. S H Saeed proposed the plan of speedy gliding factor multiplier[7] the use of the calculations of antiquated Indian vedic mathematics. IEEE 754 famous gliding element multiplier grow to be established the use of vedic era. It takes out superfluous increase ventures with zeros, empowers the parallel age of middle of the street gadgets and scaled to higher piece levels utilizing Karatsuba sutra. Proposed multiplier became planned making use of Xilinx ISE device and reproduced the use of Modelsim10.2a, directed on Spartan3E. The spread deferral inside the improved vedic coasting element multiplier have become 4.788ns. Creators inferred that form unpredictability of proposed multiplier receives diminished for contributions of large variety of bits and measured quality gets extended. usage of Urdhva Triyagbhyam sutra reduced the device necessities and deferral for skimming detail multipliers. The proposed multiplier suggests progressed expertise as some distance as pace.

Irine Padma B T, Suchitra good enough portrayed the plan and execution of IEEE754 pipelined 32-piece gliding point multiplier[8] dependent on Urdhva Triyagbhyam sutra of vedic technological know-how. The proposed plan turn out to be actualized making use of VHDL and orchestrated for a Xilinx ISE12.1. The vedic growth approach have become picked for the execution of mantissa figuring unit. the example estimation unit changed into actualized using 8-piece Ripple deliver Adder. This art work infers that, idea of pipelining diminishes the amount of LUT's which decreases the device prerequisite.

Arish S, R adequate Sharma[9] proposed a efficient technique for IEEE 754 drifting point(FP) duplication with higher deferral and electricity. The proposed multiplier utilizes the combination of Karatsuba and Urdhva Triyagbhyam calculations of vedic era. a mixture of Karatsuba and Urdhva Triyagbhyam calculations have become carried out to actualize unsigned double augmentation for mantissa depend. Proposed multiplier changed into actualized using Verilog HDL, directed on Spartan-3E and Vertex-four FPGA. The creators reasoned that the proposed form correctly dwindled the location and 
charge increment in do away with and of a FP multiplier.

ok Veeraraju, B Sujatha proposed the usage of a advanced 32-piece coasting factor multiplier using quick multipliers and adders[10]. The proposed IEEE 754 preferred gliding factor multiplier end up actualized in Verilog HDL utilising Xilinx10.1 ISE venture tool and targeted to Vertex5 FPGA. Urdhva Triyagbhyam sutra of vedic mathematics changed into implemented to form mantissa rely of drifting factor multiplier alternative in assessment to the Wallace tree multiplier. The proposed form showed a whole lot less postponement.

I V Vaibhav, ok V Saicharan, B Sravanthi, D Srinivasulu[11] exhibited IEEE 754 good sized, 32-piece gliding issue multiplier relying on vedic calculation. Urdhva Triyagbhyam sutra of vedic arithmetic end up applied to actualize $24 \times 24$ piece mantissa multiplier. The multiplier changed into deliberate in Verilog HDL and mimicked the usage of I-take a look at system. The plan became combined utilizing Xilinx12.1 tool focusing on the Xilinx Spartan3E FPGA. The proposed form used lesser considerable style of LUT's, which reduced the gadget necessity there via faded the electricity usage.

Kusuma Keerti proposed the plan of unmarried accuracy coasting factor multiplier[12] utilizing brief multipliers and quick adders. Vedic augmentation modified into implemented for mantissa duplication unit and observed to have $33 \%$ decrease in entryway encompass and $25 \%$ development in velocity whilst contrasted with skimming difficulty multipliers hooked up using Wallace tree with actualized in VHDL using Xilinx10.1 ISE duplicate equipment centered to Vertex4 FPGA with speed evaluation of - 12.

Ajay A Raut, Dr. Pravin. ok. Dahole displayed the plan of IEEE 754 single exactness skimming element multiplier using vertically and transversely set of rules[13]. Proposed configuration modified into actualized in Verilog HDL and focused on Vertex5 FPGA in Xilinx13.1 ISE programming. The contributions to the multiplier changed into 32-piece coasting point numbers and end result become sixty 4-bits on the way to improve the blunder. Reenactment outcomes display that use of variety of LUT's was 1083 and range of strengthened IOB's modified into 128.

Table I: Performance analysis \& Results

\begin{tabular}{|c|c|c|c|c|}
\hline Paper & $\begin{array}{c}\text { Number } \\
\text { of Slices }\end{array}$ & LUT's & $\begin{array}{c}\text { Bonded } \\
\text { IOB's }\end{array}$ & $\begin{array}{c}\text { Time } \\
\text { delay(ns) }\end{array}$ \\
\hline$[1]$ & 911 & 1580 & 96 & 71.293 \\
\hline$[3]$ & - & 966 & 99 & 5.246 \\
\hline$[4]$ & - & 705 & 96 & 21.823 \\
\hline$[6]$ & - & 672 & 96 & 4.94 \\
\hline$[9]$ & 1389 & 1545 & 129 & 13.141 \\
\hline$[10]$ & 2041 & 3317 & 206 & 89.374 \\
\hline$[11]$ & - & 1032 & 99 & 5.246 \\
\hline$[12]$ & 999 & 1819 & - & 14.17 \\
\hline
\end{tabular}

Table I shows the performance comparison of the 32-bit floating point multipliers of different literatures in terms of number of slices, LUT's, IOB's and time delay. Kogge-stone viper. Proposed configuration have grow to be

\section{Slices}

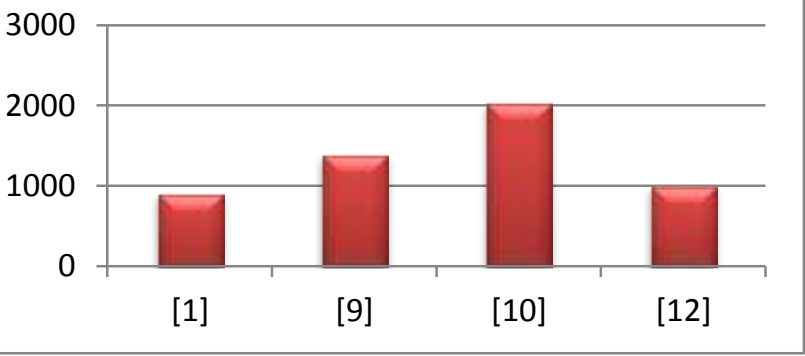

Fig.4. Comparative Chart Of Number Of Slices

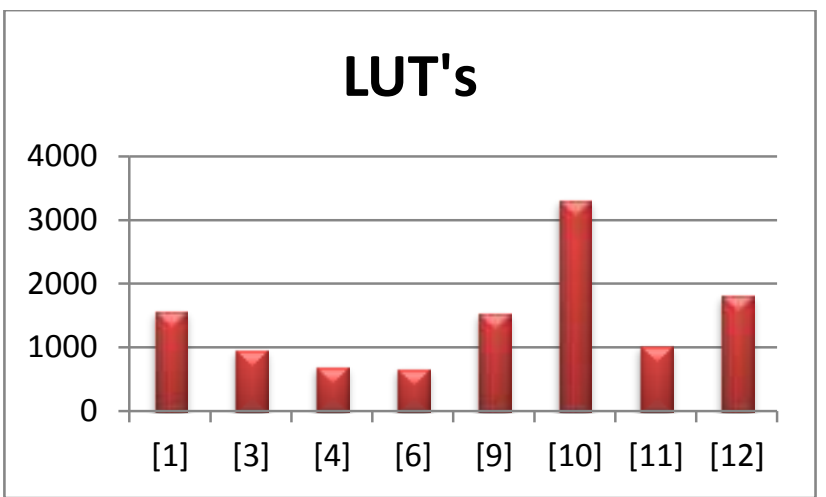

Fig.5. Comparative Chart Of Number Of LUT's

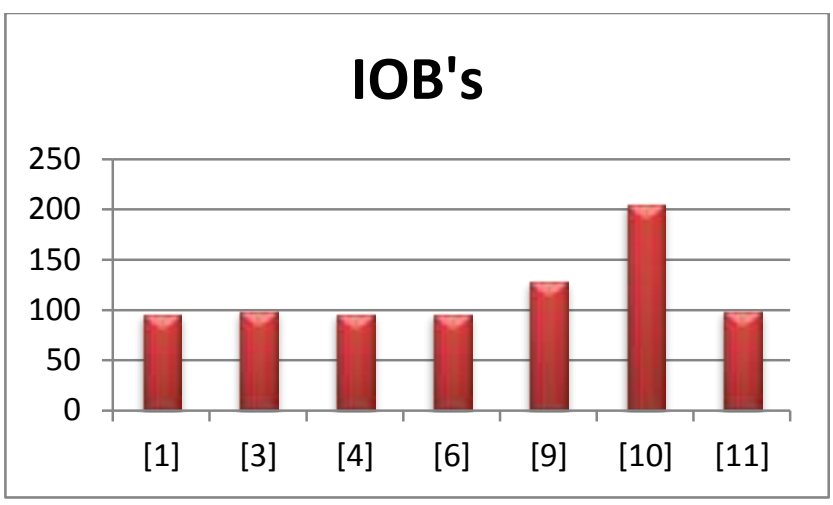

Fig.6. Comparative Chart Of IOB's

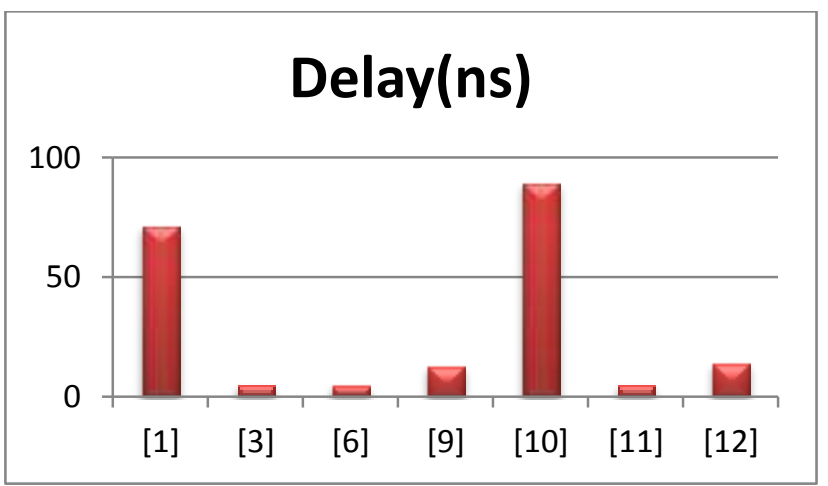

Fig.7. Comparative Chart Of Time Delay

Comparative charts in terms of number of slices, LUT's, bonded IOB's and time delay is shown in Fig. 4-7 respectively. 


\section{CONCLUSION}

IEEE 754 today's single exactness gliding factor quantity configuration contains of sign(1-bit), Mantissa(23-bits) and Exponent(eight-bits) fields. the general execution of coasting factor multiplier commonly is based totally upon the exhibition of Mantissa depend unit. Mantissa rely unit will increase unsigned parallel numbers. Urdhva Triyagbhyam sutra changed into carried out for Mantissa augmentation. unique analysts proposed their systems for gliding aspect augmentation. those structures were belief about as an prolonged way as range of cuts, LUT's, reinforced IOB's and time delays. The proposed models can be better regarding device utilization and deferral with the aid of the use of further research.

\section{REFERENCES}

1. Swapnil Suresh Mohite, Sanket Sanjay Nimbalar, Madhav Makarand Bhathande, Rashmi Rahul Kulkarni, "32 piece Floating factor Vedic Multiplier", IOSR magazine of VLSI and sign Processing(IOSR-JVSP), extent 6, problem 2, Ver. I, pp 16-20, Mar-Apr.2016.

2. Sneha khobragade, Mayur Dhait, "Plan of immoderate pace single Precision Floating factor Multiplier using Vedic arithmetic", international magazine of cutting-edge research in pc and communique Engineering, Vol.3, trouble 7, pp 6875-6882, July 2015.

3. Aniruddha kanhe, Shishir Kumar Das, Ankit Kumar Singh, "Plan and Implementation of Floating difficulty Multiplier relying on Vedic Multiplication approach", IEEE global convention on communique, records and Computing generation(ICCICT), Mumbai, pp 1-4, Oct. 19-20,2012.

4. Soumya Havaldar, k S Gurumurthy, "shape of Vedic IEEE 754 Floating point Multiplier", IEEE international conference On modern dispositions In Electronics records conversation era, pp 1131-1135, can also 20-21,2016.

5. Pooja Hatwalne, Ameya Deshmukh, Tanmay Paliwal, Krupal Lambat, " layout and Implementation of unmarried Precision Floating point Multiplier the usage of VHDL on SPARTAN three", international mag of new tendencies in Engineering and era, Vol.eight, hassle three, pp 263-269, can also additionally 2017.

6. Sushma S Mahakalkar, Sanjay L Haridas, "shape of high ordinary universal overall performance IEEE 754 Floating factor Multiplier the use of Vedic mathematics", IEEE worldwide conference on Computational Intelligence and conversation Networks, pp 985-988, 2014.

7. Pratheeksha Rai, Shailendra Kumar, Prof. S H Saeed, "structure of Floating point Multiplier the use of Vedic Aphorisms", worldwide mag of Engineering tendencies and generation(IJETT), Vol.11, amount three, pp 123-126, may additionally moreover 2014.

8. Irine Padma B T, Suchitra ok, " Pipelined Floating point Multiplier primarily based totally on Vedic Multiplication approach", worldwide magazine of contemporary studies in technological know-how, Engineering and technology, Vol.three, problem 5, pp a hundred thirty-137, July 2014.

9. Arish S, R ok Sharma, " An green Floating element Multiplier layout for excessive pace packages the use of Karatsuba set of rules and Urdhva Triyagbhyam calculation, IEEExplore, pp 303-308, July 2015.

10. proper sufficient Veeraraju, B Sujatha, "An Implementation of unmarried Precision Floating aspect Vedic Multiplier the usage of Verilog", worldwide mag of Engineering generation, control and carried out Sciences, Vol.2, hassle 7, pp 128-134, December 2014.

11. I V Vaibhav, k V Saicharan, B Sravanthi, D Srinivasulu, "VHDL Implementation of Floating factor Multiplier the usage of Vedic mathematics", international conference on electric powered powered, Electronics and Communications, pp one hundred ten-one hundred fifteen, June 2014.

12. Kusuma Keerti, "structure of immoderate regular overall performance single Precision Floating issue Multiplier", ITSI Transaction on electric and Electronics Engineering(ITSI-TEEE), Vol.2, hassle 1, pp 36-40, 2014.

13. Ajay A Raut, Dr. Pravin. excellent sufficient. Dahole, "Skimming factor Multiplier for DSP the use of Vertically and Crosswise set of policies", IETE 40 6th Mid time period Symposium "impact of technology on Sill improvement", international journal of Electronics, verbal exchange and gentle Computing generation and Engineering, pp 267-271, 2015.

14. Shweta Agrawal, Vijay Kumar Magraiya, Abhay Khedkar "usage of Vedic Multiplier on Circuit degree", global magazine of superior Engineering studies and technological statistics (IJAERS), quantity 1-No.6, pp fifty three-fifty five, 2014. 